\title{
ABSOLUTE-VALUED ALGEBRAIC ALGEBRAS
}

\section{A. A. ALBERT}

1. Introduction. An algebra $\mathfrak{A}$ over a field $\mathfrak{F}$ is a vector space over $\mathfrak{F}$ which is closed with respect to a product $x y$ which is linear in both $x$ and $y$. The product is not necessarily associative. Every element $x$ of $\mathfrak{A}$ generates a subalgebra $\mathfrak{F}[x]$ of $\mathfrak{A}$ and we call $\mathfrak{A}$ an algebraic algebra if every $\mathfrak{F}[x]$ is a finite-dimensional vector space over $\mathfrak{F}$.

We have shown elsewhere ${ }^{1}$ that every absolute-valued real finitedimensional algebra has dimension 1, 2, 4, or 8 and is either the field $\Re$ of all real numbers, the complex field $\mathfrak{E}$, the real quaternion algebra $\mathfrak{Q}$, the real Cayley algebra $\mathfrak{D}$, or certain isotopes without unity quantities of $\mathfrak{Q}$ and $\mathfrak{D}$. In the present paper we shall extend these results to algebraic algebras over $\Re$ showing that every algebraic algebra over $\Re$ with a unity quantity is finite-dimensional and so is one of the algebras listed above. The results are extended immediately to absolute-valued algebraic division algebras, that is, to algebras without unity quantities whose nonzero quantities form a quasigroup.

2. Quadratic algebras. Let $\mathfrak{F}$ be a field whose characteristic is not two and $\mathfrak{A}$ be an algebra over $\mathfrak{F}$ with a unity quantity 1 . The scalar multiples $\alpha 1$ defined for $\alpha$ in $\mathfrak{F}$ form a subalgebra of $\mathfrak{A}$ isomorphic to $\mathfrak{F}$ and we may assume that $\mathfrak{F}$ is actually a subalgebra of $\mathfrak{A}$ whose unity element coincides with that of $\mathfrak{A}$. Then $\mathfrak{F}$ is a subalgebra of the center $^{2}$ of $\mathfrak{A}$. We shall call the elements of $\mathfrak{A}$ which are in $\mathfrak{F}$ the scalars of $\mathfrak{A}$ and all other elements of $\mathfrak{A}$ nonscalars.

In an algebra of degree two over $\mathfrak{F}$ every $x$ is a root of an equation

$$
f(\xi, x) \equiv \xi^{2}-2 \xi \tau(x)-\nu(x)=0,
$$

where $\tau(x)$ and $\nu(x)$ are functions on $\mathfrak{A}$ to $\mathfrak{F}$. Then every nonscalar $x$ of $\mathfrak{A}$ determines a commutative associative algebra $\mathfrak{F}[x]=\mathfrak{F}+x \mathfrak{F}$ of order two over $\mathfrak{F}$. It is seen trivially that $f(\xi, x)$ is unique for every

Presented to the Society, September 10, 1948; received by the editors June 21, 1948.

${ }^{1}$ See the author's Absolute valued real algebras, Ann. of Math. vol. 48 (1947) pp. 495-501.

${ }^{2}$ The center of a finite-dimensional algebra was defined in the author's Nonassociative algebras I, Ann. of Math. vol. 43 (1942) on page 707. The same definition was given later for rings by T. Nakayama, Über einfache distributive Systeme unendlicher Range, Proc. Imp. Acad. Tokyo vol. 20 (1944) p. 62 and by N. Jacobson, Structure theory of rings without finiteness assumptions, Trans. Amer. Math. Soc. vol. 57 (1945) p. 239. 
nonscalar $x$ of $\mathfrak{A}$ and that $\mathfrak{F}[x]=\mathfrak{F}[y]=\mathfrak{F}+y \mathfrak{F}$ where $y=x-\tau(x)$, $\tau(y)=0$,

$$
y^{2}=\nu(y)=\nu(x)+[\tau(x)]^{2} .
$$

It will be convenient to call an element $y$ a square root if $y$ is a nonscalar and $\tau(y)=0$ so that $y^{2}=\nu(y)$ is in $\mathfrak{F}$.

An algebra $\mathfrak{A}$ will be called a quadratic algebra if $\mathfrak{A}$ has a unity quantity and $\mathfrak{F}[x]$ is a quadratic field over $\mathfrak{F}$ for every nonscalar $x$ of $\mathfrak{A}$. Then $\mathfrak{A}$ has degree two over $\mathfrak{F}$ and every $f(\xi, x)$ is irreducible for $x$ a nonscalar, $\nu(x) \neq 0$. Conversely an algebra $\mathfrak{A}$ with a unity quantity and degree two over $\mathfrak{F}$ is a quadratic algebra provided that $\nu(x) \neq 0$ for every nonscalar $x$. For if $f(\xi, x)$ were reducible we would have $f(\xi, x)=(\xi-\alpha)(\xi-\beta), y(y-\beta+\alpha)=0$ for $y=x-\alpha, y$ is a nonscalar such that $\nu(y)=0$. Assume throughout the remainder of this section that $\mathfrak{A}$ is a quadratic algebra.

The following lemma is due to L. E. Dickson. ${ }^{3}$

LEMMA 1. Let $u$ and $v$ be square roots in $\mathfrak{A}$ such that $1, u, v$ are linearly independent in $\mathfrak{F}$. Then $u v+v u$ is a scalar.

The proof is made by the computations $u^{2}=\alpha, v^{2}=\beta,(u+v)^{2}$ $=\lambda(u+v)+\mu,(u+2 v)^{2}=\rho(u+2 v)+\sigma$ for $\alpha, \beta, \lambda, \mu, \rho, \sigma$ in $\mathfrak{F}$. Then $t=u v+v u=\lambda(u+v)+\mu-(\alpha+\beta), \quad 2 t=\rho(u+2 v)+\sigma-\alpha-4 \beta$ so that $2 \lambda=\rho=2 \rho, \lambda=0, u v+v u=\mu-(\alpha+\beta)$ is in $\mathfrak{F}$.

Two nonzero elements $u$ and $v$ of $\mathfrak{A}$ are said to be J-orthogonal if their Jordan product $u v+v u=0$. We call $u_{1}, \cdots, u_{n}$ pairwise $J$ orthogonal when $u_{i}$ and $u_{j}$ are $J$-orthogonal for every $i \neq j$. Then we have

Lemma 2. Let $u_{1}, \cdots, u_{n}$ be pairwise $J$-orthogonal nonscalar elements of a quadratic algebra $\mathfrak{A}$. Then $1, u_{1}, \cdots, u_{n}$ are linearly independent in $\mathfrak{F}$.

For if $t=\lambda_{1} u_{1}+\cdots+\lambda_{n} u_{n}+\lambda_{0}=0$ for $\lambda_{0}, \lambda_{1}, \cdots, \lambda_{n}$ in $\mathfrak{F}$ then $t u_{i}+u_{i} t=2\left(\lambda_{0} u_{i}+\lambda_{i} u_{i}^{2}\right)=0$. If $\lambda_{i} \neq 0$ we would have $\nu\left(u_{i}\right)=0$ contrary to our hypothesis on $\mathfrak{A}$. Hence $\lambda_{i}=0, \lambda_{0}=0$ as desired.

Lemma 3. Let $u_{1}, \cdots, u_{n}$ be pairwise J-orthogonal square roots of $\mathfrak{A}$ and $w$ be an element of $\mathfrak{A}$ not in $\mathfrak{B}=\mathfrak{F}+u_{1} \mathfrak{F}+\cdots+u_{n} \mathfrak{F}$. Then there exists an element $b$ of $\mathfrak{B}$ such that $u=w-b$ is a square root of $\mathfrak{A}$ and $u_{1}, \cdots, u_{n}, u$ are pairwise $J$-orthogonal.

${ }^{3} \mathrm{Cf}$. Linear algebras with associativity not assumed, Duke Math. J. vol. 1 (1935) pp. 113-125. 
We have $w^{2}=2 \beta w+\gamma$ for $\beta$ and $\gamma$ in $\mathfrak{F}$ and we put $v=w-\beta$ and see that $v^{2}=\gamma+\beta^{2}, v$ is a square root. Since $v$ is not in $\mathfrak{B}$ the quantities $1, u_{i}, v$ are linearly independent and we apply Lemma 1 to obtain $v u_{i}+u_{i} v=\delta_{i}$ in $\mathfrak{F}$. Define $b_{0}=2^{-1} \sum_{j=1}^{n} \alpha_{j}^{-1} \delta_{j} u_{j}$ where $u_{j}^{2}=\alpha_{j}$. Then $b_{0} u_{i}$ $+u_{i} b_{0}=\alpha_{i}^{-1} \delta_{i} u_{i}^{2}=\delta_{i}$. But then $\left(v-b_{0}\right) u_{i}+u_{i}\left(v-b_{0}\right)=0$ and $u_{1}, \cdots, u_{n}$, $u$ are pairwise $J$-orthogonal if $u=v-b_{0}=w-b$ where $b=\beta+b_{0}$ is in $\mathfrak{B}$. Also $u^{2}=v^{2}+2^{-1} \sum_{j=1}^{n} \alpha_{j}^{-1} \delta_{j}\left(v u_{j}+u_{j} v\right)+4^{-1} \sum_{j=1}^{n} \alpha_{j}^{-2} \delta_{j}^{2}$ is in $\mathfrak{F}$ and our proof is complete.

We shall apply the simple results just obtained to prove the finite dimensionality of alternative quadratic algebras, that is, of quadratic algebras such that $x(x y)=(x x) y,(y x) x=y(x x)$ for every $x$ and $y$ of $\mathfrak{A}$. It is necessary to consider only the case where $\mathfrak{A}$ contains a subspace $\mathfrak{S}$ of dimension at least eight. Thus we may assume that $\mathfrak{A}$ contains a pair of $J$-orthogonal square roots $u$ and $v$ such that $u^{2}=\alpha \neq 0$ in $\mathfrak{F}, v^{2}=\beta \neq 0$ in $\mathfrak{F}, u v=-v u$. By the alternative law we have

$$
u(u v)=\alpha v,(u v) u=-(v u) u=-v(u u)=-\alpha v \neq 0 .
$$

Then $u v$ and $u$ are $J$-orthogonal. Similarly $u v$ and $v$ are $J$-orthogonal. But then $u v \neq 0$ is a nonscalar and, by Lemma $2, \mathfrak{A}$ contains the fourdimensional subspace $\mathfrak{Q}=\mathfrak{F}+u \mathfrak{F}+v \mathfrak{F}+u v \mathfrak{F}$ of $\mathfrak{A}$. Also $(u v)^{2}=\gamma+\delta u v$ for $\gamma$ and $\delta$ in $\mathfrak{F}, u\left[(u v)^{2}\right]=[u(u v)](u v)=\alpha v(u v)=-\alpha v(v u)=-\alpha \beta u$ $=\gamma u+\alpha \delta v$. It follows that $\alpha \delta=0$ and so $(u v)^{2}=\gamma=-\alpha \beta$. Evidently $\mathfrak{Q}$ is a linear algebra which is a subring of $\mathfrak{A}$ and it is easily verified, since all products of basal elements of $\mathfrak{Q}$ are now known, that $\mathfrak{Q}$ is an associative generalized quaternion algebra. We state the result as

LEMмA 4. Let $u$ and $v$ be J-orthogonal square roots of an alternative quadratic algebra $\mathfrak{A}$. Then $\mathfrak{O}=\mathfrak{F}+u \mathfrak{F}+v \mathfrak{F}+u v \mathfrak{F}$ is a four-dimensional subring of $\mathfrak{A}$ and is a generalized quaternion associative algebra with $u^{2}=\alpha, v^{2}=\beta,(u v)^{2}=-\alpha \beta, v u=-u v$ for $\alpha \neq 0, \beta \neq 0$ in $\mathfrak{F}$.

Assume next that $\mathfrak{A}$ contains $\mathfrak{Q}$ as well as an element not in $\mathfrak{Q}$. Then Lemma 3 implies that there exists a square root which is $J$-orthogonal to $u, v$ and $u v$. For every such square root we prove

LEMMA 5. Let $u_{1}=u, u_{2}=v, u_{3}=u v, u_{4}=w$ be a set of pairwise $J$ orthogonal square roots of an alternative quadratic algebra $\mathfrak{A}$. Then $u_{1}, \cdots, u_{4}, u_{5}=u w, u_{6}=v w, u_{7}=(u v) w$ are pairwise J-orthogonal, $u_{i}^{2}=\alpha_{i}$ in $\mathfrak{F}, u_{i} u_{j}=\alpha_{i j} u_{k}$ for every $i \neq j$ where $\alpha_{i j}$ is in $\mathfrak{F}$ and $k$ depends on $i$ and $j$. Also $\mathfrak{B}=\mathfrak{F}+u_{1} \mathfrak{F}+\cdots+u_{7} \mathfrak{F}$ is an eight-dimensional subalgebra of $\mathfrak{A}$.

For let $\alpha=\alpha_{1}, \beta=\alpha_{2}, \gamma=\alpha_{4}$. Then $(u+w)^{2}=\alpha+\gamma$ and by the alternative law $(\alpha+\gamma) v=(u+w)^{2} v=(u+w)(u v+w v)=u(u v)+w(u v)$ 
$+u(w v)+w(w v)=\alpha v+\gamma v-u(v w)-(u v) w$. Thus

$$
u(v w)=-(u v) w .
$$

Similarly $(\alpha+\gamma) v=v(u+w)^{2}=(v u+v w)(u+w)=(v u) u+(v w) w+(v u) w$ $+(v w) u,(v w) u=-(v u) w$,

$$
(v w) u=(u v) w=-u(v w) .
$$

We have proved that $u$ and $v w$ are $J$-orthogonal. By symmetry $v$ is $J$-orthogonal to $u w$ and

$$
v(u w)=-(u w) v=(u v) w .
$$

However we may replace $u, v$ by $u v, v$ to see that $v w$ and $u v$ are $J$-orthogonal. Also $\mathfrak{F}+v \mathfrak{F}+w \mathfrak{F}+v w \mathfrak{F}$ is a quaternion algebra and $(v w)^{2}=-\beta \gamma$, vw and $v$ are $J$-orthogonal.

Formula (4) may be applied with $u$ and $v$ replaced by $u v$ and $u$ to yield

$$
(u w)(u v)=[(u v) u] w=-\alpha v w .
$$

Also $(u w) v=(v u) w=-(u v) w$. Then $(u+u w)^{2}(u v)=(\alpha-\alpha \gamma) u v$ $=(u+u w)[(u+u w)(u v)]=(u+u w)(\alpha v-\alpha v w)=\alpha u v+\alpha(u w) v-\alpha u(v w)$ $-\alpha(u w)(v w)$. But $\alpha(u w) v-\alpha u(v w)=-\alpha(u v) w+\alpha(u v) w=0$. Hence

$$
(u w)(v w)=\gamma u v \text {. }
$$

Interchange $u$ and $v$ to obtain $(v w)(u w)=\gamma v u=-(u w)(v w)$. It follows immediately that the symmetry among the elements $u, v, u v$ implies that the elements $u_{1}, \cdots, u_{7}$ are pairwise $J$-orthogonal. They are square roots since a product such as $u w$ is in a quaternion algebra $\mathfrak{F}+u \mathfrak{F}+w \mathfrak{F}+u w \mathfrak{F}$ and is a square root by Lemma 4 . But (4) and (7) yield $u_{i} u_{j}=\alpha_{i j} u_{k}$.

We finally assume that $\mathfrak{A}$ contains $\mathfrak{B}=\mathfrak{F}+u_{1} \mathfrak{F}+\cdots+u_{7} \mathfrak{F}$ and an element not in $\mathfrak{B}$. Then $\mathfrak{A}$ contains an element $z$ which is $J$-orthogonal to $u_{1}, \cdots, u_{7}$. We form the product $t=(u v)(w z)$. Now $z^{2}=\delta \neq 0$ in F, $(u v) t=-\alpha \beta w z,[(u v) t]^{2}=\alpha \beta \gamma \delta \neq 0, t \neq 0$. However we use the quaternion algebra $\mathfrak{F}+u v \mathfrak{F}+w \mathfrak{F}+(u v) w \mathfrak{F}$ with $z$ and apply (4) to obtain $t=-[(u v) w] z$. Then $t=[u(v w)] z$. Since $z$ is orthogonal to $u$, $v w$ and $u(v w)$ we now have $t=-u[(v w) z]=u[v(w z)]$. Apply Lemma 5 with $u_{1}=u, u_{2}=w, u_{4}=z$ to get $w z$ orthogonal to $u$. The same values, except for $u_{1}=v$, yields $w z$ orthogonal to $v$ and similarly $u_{1}=u v$ implies that $w z$ is orthogonal to $u v$. But then (4) yields $t=-(u v)(w z)$ $=-t$. We have proved ${ }^{4}$

${ }^{4}$ Alternative quadratic algebras of finite dimension were determined by M. Zorn, Abh. Math. Sem. Hamburgischen Univ. vol. 9 (1933) pp. 395-402. 
THEOREM 1. Every alternative quadratic algebra is an algebra of order $n=1,2,4,8$ over $\mathfrak{F}$.

3. Absolute valued algebraic algebras. Let $\Re$ be the field of all real numbers and $\mathfrak{A}$ be an algebraic algebra over $\Re$. We assume the existence of a function $|a|$ on $\mathfrak{A}$ to $\Re$ such that $|0|=0,|a|>0$ if $a \neq 0,|a b|=|a||b|,|a+b| \leqq|a|+|b|$ for every $a$ and $b$ of $\mathfrak{A}$. Assume also that if $a$ is in $\mathfrak{A}$ and $\alpha$ is any real number then $|\alpha a|=|\alpha||a|$ where $|\alpha|$ is the ordinary absolute value of $\alpha$. Then we call $\mathfrak{A}$ an absolute-valued algebra.

We shall assume that $\mathfrak{A}$ has a unity quantity 1 so that $\Re$ may be taken to be a subalgebra of $\mathfrak{A}$ and the hypothesis $|\alpha a|=|\alpha||a|$ now becomes simply the hypothesis that $|a|$ is the ordinary absolute value whenever $a$ is a real number. The hypothesis that $\mathfrak{A}$ is an algebra over $\Re$ implies that $\Re[x]$ is an absolute-valued real algebra generated by $x$ and containing 1 . However all real, absolute-valued algebras with a unity quantity are known, ${ }^{1}$ they all have degree one or two and hence $\Re[x]$ is isomorphic to either $\Re$ or the field $\mathbb{C}$ of all complex numbers. It follows immediately that $\mathfrak{A}$ is a quadratic algebra over $\Re$.

Every element $x$ of $\mathfrak{A}$ now has the form $x=\alpha+\beta u$ where $\alpha$ and $\beta$ are real and $u^{2}=-1$. Then $\Re(u)$ is isomorphic to $\mathbb{E},(x-\alpha)^{2}+\beta^{2}=0$, the roots of $f(\xi, x)=\xi^{2}-2 \alpha \xi+\alpha^{2}+\beta^{2}$ are $\rho, \bar{\rho}$ where $\rho=\alpha+\beta i, \bar{\rho}=\alpha$ $-\beta i, \rho \bar{\rho}=\alpha^{2}+\beta^{2},|\rho|=\left(\alpha^{2}+\beta^{2}\right)^{1 / 2}$. It is known ${ }^{3}$ that the minimum function of $x$ and $R_{x}$ coincide with $f(\xi, x)$ and that $|x|=|\rho|$ $=\left(\alpha^{2}+\beta^{2}\right)^{1 / 2}$.

Assume that $u_{1}, \cdots, u_{n}$ are pairwise $J$-orthogonal quantities such that $u_{i}^{2}=-1$. Then if $x=\alpha_{0}+\alpha_{1} u_{1}+\cdots+\alpha_{n} u_{n}$ we have $x^{2}-2 \alpha_{0} x$ $+\alpha_{0}^{2}+\cdots+\alpha_{n}^{2}=0$. It follows that $|x|^{2}=\alpha_{0}^{2}+\alpha_{1}^{2}+\cdots+\alpha_{n}^{2}$.

We are now able to compute the absolute values of quantities and shall assume that $\mathfrak{A}$ contains a pair of $J$-orthogonal quantities $u$ and $v$ such that $u^{2}=v^{2}=-1$. By Lemma 3 we may write $u v=\lambda_{1}+\lambda_{2} u$ $+\lambda_{3} v+\lambda_{4} w$ where $\lambda_{1}, \cdots, \lambda_{4}$ are real, $u, v, w$ are pairwise $J$-orthogonal and $w^{2}=-1$. Of course when $u v$ is in $\mathfrak{F}+u \mathfrak{F}+v \mathfrak{F}$ this will be given by $\lambda_{4}=0$. Then $|u v|^{2}=\left.\left.|u|^{2}\right|^{v}\right|^{2}=1=\lambda_{1}^{2}+\lambda_{2}^{2}+\lambda_{3}^{2}+\lambda_{4}^{2}$. But $|1+v|^{2}=2,|u(1+v)|^{2}=2=|u+u v|^{2}=\left|\lambda_{1}+\left(\lambda_{2}+1\right) u+\lambda_{3} v+\lambda_{4} v\right|^{2}=\lambda_{1}^{2}$ $+\lambda_{2}^{2}+\lambda_{3}^{2}+\lambda_{4}^{2}+1+2 \lambda_{2}$. Then $\lambda_{2}=0$. A similar computation of $|(1+u) v|^{2}$ yields $\lambda_{3}=0, \lambda_{1}^{2}+\lambda_{4}^{2}=1$. Finally $|(1+u)(1+v)|^{2}=\mid\left(\lambda_{1}+1\right)$ $+u+v+\left.\lambda_{4} v\right|^{2}=4=\lambda_{1}^{2}+\lambda_{4}^{2}+3+2 \lambda_{1}, \lambda_{1}=0, \lambda_{4}= \pm 1, u v= \pm w$ is $J$ orthogonal to $u$ and $v$ and $(u v)^{2}=w^{2}=-1$.

Apply the argument above to the $J$-orthogonal quantities $u$ and $u v$ to see that $[u(u v)]^{2}=-1$ and that $u(u v)$ is $J$-orthogonal to both $u$ and $u v$. Then $u(u v)=\xi v+\eta z$ where $\xi$ and $\eta$ are real, $z$ is $J$-orthogonal to 
$u, v$ and $u v, z^{2}=-1$ and of course the nonexistence of such a $z$ implies that $\eta=0$. Since $|u(w v)|=|u|^{2}|v|=1$ we have $\xi^{2}+\eta^{2}=1$. We form the product $(-1+u)(v+u v)=-v+u v-u v+u(u v)=-v+u(u v)$ $=(\xi-1) v+\eta z$ and see that $4=|(\xi-1) v+\eta z|^{2}=\xi^{2}+\eta^{2}+1-2 \xi,-2 \xi$ $=2, \xi=-1, \xi^{2}+\eta^{2}=1+\eta^{2}=1, \eta=0$. Hence $u(u v)=-v$.

The results above imply that $\mathfrak{A}$ is alternative. Indeed the relation $x(x y)=(x x) y$ is equivalent to $(\alpha+\beta u)[(\alpha+\beta u) y]=[(\alpha+\beta u)(\alpha+\beta u)] y$ for real $\alpha$ and $\beta$ where $u^{2}=-1$. But $\alpha(\alpha y)+(\beta u) \alpha y+\alpha[(\beta u) y]$ $=\alpha^{2} y+[(\alpha \beta) u] y+[(\beta u) \alpha] y$ and so we need only prove $u(u y)=(u u) y$. We may also write $y=\lambda+\mu u+\nu v$ where $\lambda, \mu, \nu$ are real, $u v=-v u$ and $v^{2}=-1$, and the linearity of our relation in $y$ implies that we need only prove the trivial $u\left(u^{2}\right)=u^{2} u=-u$ and the relation $u(u v)=(u u) v$ $=-v$ proved above. Similarly the relation $(y x) x=y(x x)$ is implied by $(v u) u=-(u v) u=u(u v)=-v=v(u u)$. We then apply Theorem 1 to obtain

THEOREM 2. Let $\mathfrak{A}$ be an absolute-valued real algebraic algebra with a unity quantity. Then $\mathfrak{A}$ is a finite-dimensional algebra and is indeed the field $\mathfrak{R}$ or $\mathfrak{S}$, the quaternion algebra $\mathfrak{Q}$ or the Cayley algebra $\mathfrak{D}$.

An algebra is called a division algebra if its nonzero elements form a quasigroup. For such algebras we have

THEOREM 3. Let $\mathfrak{A}$ be an absolute valued real algebraic division algebra without a unity element. Then $\mathfrak{A}$ is an algebra of order four or eight.

For the mappings $x \rightarrow x g=x R_{g}$ and $x \rightarrow g x=x L_{g}$ are one-to-one mappings of $\mathfrak{A}$ on $\mathfrak{A}$ for every $g \neq 0$ of $\mathfrak{A}$ and so $R_{g}$ and $L_{o}$ have inverses. Select $g$ so that $|g|=1$ and take $P=R_{g}^{-1}, Q=L_{g}^{-1}$. Let $\mathfrak{A}_{0}$ be the same real vector space as $\mathfrak{A}$ and define multiplication in $\mathfrak{A}_{0}$ by $x \cdot y=(x P)(y Q)$. Then if $e=g g$ we have $e P=e Q=g, e \cdot y=g(y Q)$ $=y L_{0}^{-1} L_{o}=y, y \cdot e=\left(y R_{g}^{-1}\right) g=y$ and so $\mathfrak{N}_{0}$ has $e$ as its unity quantity. Since $\mathfrak{A}_{0}$ is the same vector space as $\mathfrak{A}$ the additive and scalar properties of $|x|$ all hold in $\mathfrak{A}_{0 .}$ But $|x \cdot y|=|x P| \cdot|y Q|=|x||y|$ since $(x P) g=x, \quad|x|=|x P||g|=|x P|, \quad g(y Q)=y, \quad|y|=|g||y Q|=|y Q|$. Thus $\mathfrak{A}_{0}$ is an absolute valued algebraic algebra with a unity quantity and is finite-dimensional by Theorem $2, \mathfrak{A}$ is finite-dimensional. However the result on the dimension of $\mathfrak{A}$ of our Theorem is known ${ }^{1}$ for algebras.

The University of Chicago 